(2) Open Access Full Text Article

\title{
Serum Hypoxia-Inducible Factor Ialpha Levels Correlate with Outcomes After Intracerebral Hemorrhage
}

\author{
Yong Cai' \\ Yao-Kun Zhuang' \\ Xiao-Yu Wu' \\ Xiao-Qiao Dong ${ }^{2}$ \\ Quan $\mathrm{Du}^{2}$ \\ Wen-Hua $\mathrm{Yu}^{2}$ \\ Ke-Yi Wang ${ }^{2}$ \\ Wei $\mathrm{Hu}^{3}$ \\ Yong-Ke Zheng ${ }^{3}$
}

'The Fourth School of Clinical Medicine, Zhejiang Chinese Medical University, Hangzhou, 3I0053, Zhejiang Province,

People's Republic of China; ${ }^{2}$ Department of Neurosurgery, Affiliated Hangzhou First People's Hospital, Zhejiang University School of Medicine, Hangzhou, 310006, Zhejiang Province, People's Republic of China; ${ }^{3}$ Department of Intensive Care Unit, Affiliated Hangzhou First People's Hospital, Zhejiang University School of Medicine, Hangzhou, 3l0006, Zhejiang Province, People's Republic of China
Correspondence: Xiao-Qiao Dong Department of Neurosurgery, Affiliated Hangzhou First People's Hospital,

Zhejiang University School of Medicine,

26I Huansha Road, Hangzhou, 310006,

Zhejiang Province, People's Republic of

China

Email dxqhyy@I63.com
Background: Serum hypoxia-inducible factor lalpha (HIF-1 $\alpha$ ) is a key regulator in hypoxic and ischemic brain injury. We determined the relationship between serum HIF-1 $\alpha$ levels and long-term prognosis plus severity of intracerebral hemorrhage (ICH).

Methods: A total of $97 \mathrm{ICH}$ cases and 97 healthy controls were enrolled. Glasgow Coma Scale (GCS) score and hematoma volume were used to assess hemorrhagic severity. Glasgow Outcome Scale (GOS) score of 1-3 at post-stroke 90 days was defined as a poor outcome.

Results: Serum HIF-1 $\alpha$ levels of ICH patients were significantly higher than those of healthy controls (median, 218.8 vs $105.4 \mathrm{pg} / \mathrm{mL} ; \mathrm{P}<0.001$ ) and were substantially correlated with GCS score $(r=-0.485, \mathrm{P}<0.001)$, hematoma volume $(r=0.357, \mathrm{P}<0.001)$ and GOS score $(r=-0.436, \mathrm{P}<0.001)$. Serum HIF-1 $\alpha$ levels $>239.4 \mathrm{pg} / \mathrm{mL}$ discriminated patients at risk of 90 -day poor outcome with sensitivity of $65.9 \%$ and specificity of $79.3 \%$ (area under the receiver operating characteristic curve, $0.725 ; 95 \%$ confidence interval, 0.625-0.811; $\mathrm{P}<0.001$ ). Moreover, serum HIF-1 $\alpha$ levels $>239.4 \mathrm{pg} / \mathrm{mL}$ were independently associated with a poor 90-day outcome (odds ratio, 5.133; 95\% confidence interval, 1.117-23.593; $\mathrm{P}=0.036)$.

Conclusion: Serum HIF-1 $\alpha$, in close correlation with hemorrhagic severity and poor 90 -day outcome, may serve as a potential prognostic biomarker for ICH.

Keywords: intracerebral hemorrhage, prognosis, severity, hypoxia-inducible factor 1alpha

\section{Introduction}

Spontaneous intracerebral hemorrhage (ICH) is a common cerebrovascular disease and represents one of the major leading causes of death and long-term disability worldwide. ${ }^{1}$ Hypoxic and ischemic brain injury is a very important process implicated in pathophysiological mechanisms of secondary brain injury after $\mathrm{ICH}$, finally leading to neuronal death and inducing neurologic dysfunction. ${ }^{2,3}$ Besides clinical and radiological parameters, biochemical variables have been noted for early prediction of ICH prognosis in recent decades. ${ }^{4}$ Hypoxia-inducible factor-1 (HIF-1) is identified as a master regulator of hypoxia-responsive genes. ${ }^{5}$ It regulates the expression of a broad range of genes that facilitate adaptation to low oxygen conditions. ${ }^{6}$ It has two protein subunits, among which, one is HIF-1alpha (HIF-1 $\alpha$ ) and the other is HIF1beta (HIF-1 $\beta){ }^{7}$ Reportedly, HIF-1 $\alpha$ consists in neuronal cells ${ }^{8}$ and primary cortical astrocytes, ${ }^{9}$ as well as its expression was greatly up-regulated in brain tissue after experimental $\mathrm{ICH}^{10}$ Clearly, HIF-1 $\alpha$ plays an essential role in the fate of injured neurons after hemorrhagic, traumatic or ischemic brain injury. ${ }^{11-13}$ Previous 
experiments have shown that HIF-1 $\alpha$ was implicated in neuronal apoptosis, brain edema formation and disruption of blood-brain barrier. ${ }^{11-13}$ Contrarily, some other studies demonstrated protective effect of HIF-1 $\alpha$ against neuronal apoptosis and neurologic dysfunction in vitro or in vivo. ${ }^{14-17}$ Thus, HIF-1 $\alpha$ appears to be implicated in the cell's response to brain injury with a double-edged sword effect. In a previous study, ${ }^{18}$ using univariate analysis and a small sample size (40 patients), it was shown that elevated serum HIF-1 $\alpha$ levels were correlated with cerebral infarction size after acute ischemic stroke. However, to my best knowledge, HIF-1 $\alpha$ levels have not been explored in the peripheral blood of human ICH. In the current study, we aimed to determine whether serum HIF-1 $\alpha$ could be associated with the severity and long-term prognosis of $\mathrm{ICH}$.

\section{Materials and Methods Study Population}

From January 2019 to April 2020, we performed a prospective, observational study and recruited all firstever ICH patients, who were admitted within $24 \mathrm{~h}$ after symptom onset of stroke. The exclusion criteria included 1) age less than 18 years; 2) brain hemorrhages as a result of head trauma, venous sinus thrombosis, ischemic stroke or intracranial tumors; 3) prior hemorrhagic or ischemic stroke; and 4) other diseases such as severe infection within recent 4 weeks, autoimmune diseases and known malignancies. Simultaneously, healthy subjects were selected as controls. This study was performed based on the ethical guidelines of the Declaration of Helsinki and the approval for its protocol was obtained from the Institutional Review Board of Affiliated Hangzhou First People's Hospital, Zhejiang University School of Medicine, Opinion Number: [2020] Medical Ethics Review No. (058)-01. The written informed consent was acquired from controls themselves or relatives of patients.

\section{Assessment}

We collected some related data, such as demographics, vascular risk factors, hematoma location, radiological parameters, vital signs, clinical features and medical history. Glasgow Coma Scale (GCS) was utilized to evaluate stroke severity at admission. Hematoma volume was measured in accordance with $\mathrm{ABC} / 2$ method. ${ }^{19}$ The presence of intraventricular extension of hematoma was also recorded. Non-invasive blood pressure determinations were performed as well as systolic and diastolic arterial blood pressures were recorded upon arrival at emergency center. A Glasgow Outcome Scale (GOS) score of 1-3 at 90 days after stroke onset was assessed as a poor outcome.

\section{Determinations}

Peripheral blood samples of ICH patients were drawn from venous vessels at emergency center and those of healthy controls were obtained at study entry. Afterwards, centrifugation of samples was done to remove cellular debris, and then, the supernatant was at once frozen at $-80{ }^{\circ} \mathrm{C}$ until the final measurements. Serum HIF-1 $\alpha$ concentrations were gauged by enzyme-linked immunosorbent assay with commercially available kits (RapidBio Lab, California, USA) in accordance with the manufacturer's instructions. The measurements were completed by the same technician who was inaccessible to clinical information.

\section{Statistical Analysis}

Statistical analyses were carried out with Statistical Package for the Social Sciences version 19.0. Using the Kolmogorov-Smirnov test or Shapiro-Wilk test, the normal distribution of quantitative data was assessed. Because all quantitative data were non-normally distributed, they were reported as medians with 25 th-75th percentiles. Qualitative data were reported as counts (proportions). Data between two groups were compared using the $\chi^{2}$ test, Fisher's exact test or Mann-Whitney $U$-test as appropriate. Kruskal-Wallis $H$-test was performed to compare data among multiple groups. Bivariate correlations between serum HIF-1 $\alpha$ concentrations and GCS score in addition to hematoma volume were at first analyzed using Spearman correlation coefficients. And then, multivariate linear regression model was built to discern independent correlations between serum HIF-1 $\alpha$ concentrations and GCS score plus hematoma volume after adjusting for other confounding factors, including demographics and vascular risk factors. In order to identify predictors independently associated with 90-day poor outcome, we constructed a binary logistic regression model, which contained the significant variables $(\mathrm{P}<0.05)$ in the univariate analysis. Associations were shown as odds ratio and 95\% confidence intervals. Receiver operating characteristic (ROC) curve was used to appraise the value of serum HIF-1 $\alpha$ levels in predicting the 90-day outcome after ICH and the area under the curve (AUC) was estimated. A P $<0.05$ was considered statistically significant. 


\section{Results}

\section{Patient Selection and Characteristics}

During the study period, a total of 134 first-ever spontaneous ICH patients admitted within $24 \mathrm{~h}$ after symptom onset of stroke were first analyzed. Afterwards, we removed 21 patients with secondary brain bleedings, 7 patients presenting with other above-mentioned diseases, 7 patients with previous stroke and 2 patients with loss to follow-up. At last, a total of $97 \mathrm{ICH}$ cases were eventually enrolled for further analysis. Also, 97 healthy controls were recruited. There were no differences in age and percentages of sex, current smoking and alcohol consumption between patients and controls; and patients had significantly higher proportions of hypertension and diabetes mellitus than controls (Table 1).

ICH patients consisted of 55 males and 42 females, as well as their age ranged from 31 to 90 years (median, 62 years; 25 th -75 th percentiles, 51-69 years). In the aggregate, 23 patients smoked cigarette and 32 patients consumed alcohol. History of past illness included hypertension (57 cases) and diabetes mellitus (14 cases). The patients were admitted at a median value of $6.5 \mathrm{~h}$ after symptom onset (range, 0.5-24.0 h; 25th-75th percentiles, 4.0-9.0 h), with their blood samples collected from 1.0 to $26.0 \mathrm{~h}$ (median, 9.0 $\mathrm{h} ; 25 \mathrm{th}-75$ th percentiles, 6.0-13.0 h) after stroke. As regards hemorrhagic clinical severity, the median value of GCS scores was 13 (range, 4-15; 25th-75th percentiles, 9-15). With respect to some radiological parameters, hematomas of 20 cases were located at cerebral lobe; those of 15 cases were found at infratentorial cavity; intraventricular bleedings was present in 26 cases; and the median value of hematoma volume was $14.5 \mathrm{~mL}(25 \mathrm{th}-75$ th percentiles, 6.7-30 mL; range, $2.0-64.8 \mathrm{~mL}$ ). Via non-invasive arterial pressure measurements, the median values of systolic

Table I Comparisons of Demographic Data and Vascular Risk Factors Between Controls and Patients with Acute Intracerebral Hemorrhage

\begin{tabular}{|l|c|c|c|}
\hline & Patients & Controls & \\
\hline Age (years) & $62(5 \mathrm{I}-69)$ & $59(5 \mathrm{I}-67)$ & 0.195 \\
Sex (male/female) & $55 / 42$ & $50 / 47$ & $0.47 \mathrm{I}$ \\
Hypertension & $57(58.8 \%)$ & 0 & $<0.00 \mathrm{I}$ \\
Diabetes mellitus & $14(14.4 \%)$ & 0 & $<0.00 \mathrm{I}$ \\
Current smoking & $23(23.7 \%)$ & $18(18.6 \%)$ & 0.379 \\
Alcohol consumption & $32(33.0 \%)$ & $25(25.8 \%)$ & 0.270 \\
\hline
\end{tabular}

Notes: Quantitative data were reported as medians with 25 th-75th percentiles. Qualitative data were presented as counts (proportions). Intergroup comparisons of various variables were performed using the $\chi^{2}$ test or Fisher's exact test for qualitative data, and Mann-Whitney $U$-test for quantitative data. arterial pressure and diastolic arterial pressure were 155 $\mathrm{mmHg}$ (range, 107-234 $\mathrm{mmHg} ; 25 \mathrm{th}-75$ th percentiles, 144-173 $\mathrm{mmHg}$ ) and $88 \mathrm{mmHg}$ (range, 55-188 $\mathrm{mmHg}$; 25th-75th percentiles, $78-98 \mathrm{mmHg}$ ) respectively. Laboratory tests showed that blood leucocyte count ranged from 3.4 to $19.7 \times 10^{9} / 1$, with a median value of $9.0 \times 10^{9} / 1$ (25th-75th percentiles, $\left.6.8-11.3 \times 10^{9} / 1\right)$, and there was a median value of $6.7 \mathrm{mmol} / \mathrm{l}$ at serum glucose levels (range, 2.5-16.8 mmol/1; 25th-75th percentiles, 5.3-8.9 mmol/l). At 90 days after stroke, GOS scores 1, 2, 3, 4 and 5 were found in 10,14, 20, 36 and 17 patients, respectively; and GOS score range from 1 to 5 (median, 4; 25th-75th percentiles, 3-4). According to definition of poor outcome (GOS scores 1-3), a total of 44 patients suffered from a poor outcome at 90 days after stroke.

\section{Change of Serum HIF-I $\alpha$ Levels}

Among this group of ICH patients, serum HIF-1 $\alpha$ levels ranged from 71.7 to $585.8 \mathrm{pg} / \mathrm{mL}$, with a median value of $218.8 \mathrm{pg} / \mathrm{mL}$ (25th-75th percentiles, 171.2-298.7 pg/mL). There was $105.4 \mathrm{pg} / \mathrm{mL}$ at the median value of serum HIF$1 \alpha$ levels of healthy controls (range, 39.1-192.9 pg/mL; 25th -75th percentiles, 87.0-143.7 pg/mL). Using Mann-Whitney $U$-test, it was clear that serum HIF-1 $\alpha$ levels were markedly higher in ICH patients than in healthy controls (Figure 1).

\section{Correlation of Serum HIF-I $\alpha$ Levels with Hemorrhagic Severity}

In order to verify correlations of serum HIF-1 $\alpha$ levels with hemorrhagic severity indicated by GCS score and

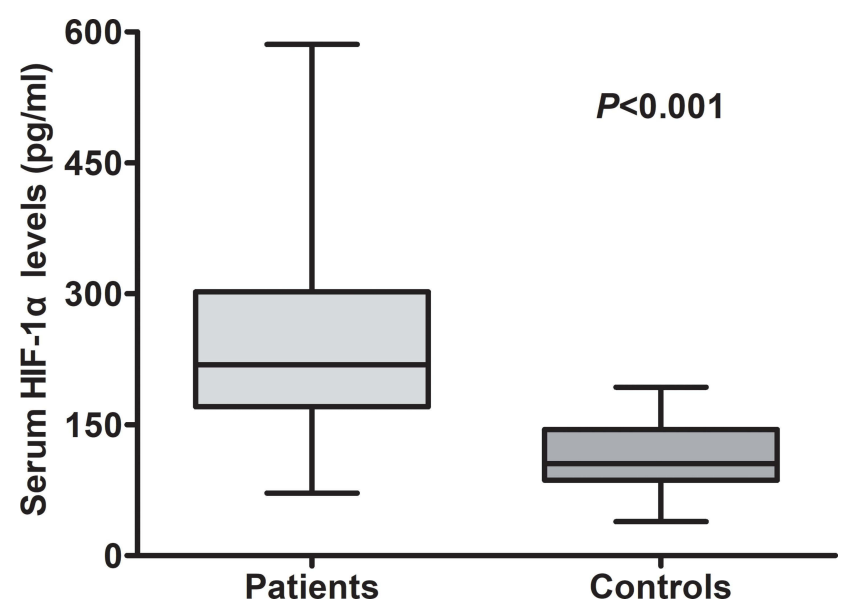

Figure I Difference of serum hypoxia-inducible factor Ialpha levels between healthy controls and patients with intracerebral hemorrhage. Using MannWhitney $U$-test, serum hypoxia-inducible factor Ialpha levels were significantly higher in intracerebral hemorrhage patients than in healthy controls $(P<0.00 \mathrm{I})$. Abbreviation: HIF-I $\alpha$, hypoxia-inducible factor Ialpha. 
hematoma volume, GCS score and hematoma volume were identified as quantitative and qualitative variables. ICH patients were divided into three subgroups in accordance with GCS score, namely GCS score 3-8, 9-12 and 13-15. In addition, ICH patients were dichotomized based on the cutoff value of $30 \mathrm{~mL}$ at hematoma volume. Figures 2 and 3 show the significant correlations existed between serum HIF- $1 \alpha$ levels and GCS score in addition to hematoma volume among this group of ICH patients. Furthermore, after controlling for demographic and vascular risk factors using multivariate analysis, their close correlations still existed (Table 2).

\section{Relationship Between Serum HIF-I $\alpha$ Levels and Outcome}

Just as displayed in Figure 4, serum HIF-1 $\alpha$ levels were remarkably declined with ascending GOS scores. In Figure 5, serum HIF-1 $\alpha$ levels were substantially higher in patients with poor outcome than in those with good outcome. Moreover, under ROC curve, serum HIF- $1 \alpha$ levels remarkably predicted post-stroke 90 -day poor outcome among this group of ICH patients (AUC, 0.725; 95\% confidence interval, 0.625-0.811); and serum HIF-1 $\alpha$ level above $239.4 \mathrm{pg} / \mathrm{mL}$ distinguished patients with development of poor 90-day outcome with specificity and sensitivity values of $79.3 \%$ and $65.9 \%$ (Youden index J, 0.452) respectively. Moreover, Figure 6 shows that its discriminatory ability was equivalent to those of GCS score (AUC, $0.792 ; 95 \%$ confidence interval, 0.697-0.868; $\mathrm{P}=0.248$ ) and hematoma volume (AUC, 0.817; 95\% confidence interval, 0.725-0.888; $\mathrm{P}=0.124)$.

Tables 3 and 4 show that, as compared to patients who had GOS score of 4-5, patients presenting with GOS score of $1-3$ had a significantly high percentage of serum HIF- $1 \alpha$ level above $239.4 \mathrm{pg} / \mathrm{mL}$, displayed the substantially elevated age, serum glucose levels and hematoma volumes, as well as exhibited a remarkably declined GCS score. The aforementioned significant variables in univariate analyses were contained in the multivariable model, and subsequently it was demonstrated that the factors independently associated with 90-day poor outcome among such a host of ICH patients were GCS score, hematoma volume and serum HIF-1 $\alpha$ level above $239.4 \mathrm{pg} / \mathrm{mL}$ (Table 5).

\section{Discussion}

Since a previous study found the intimate correlation of serum HIF-1 $\alpha$ levels with cerebral infarction size after
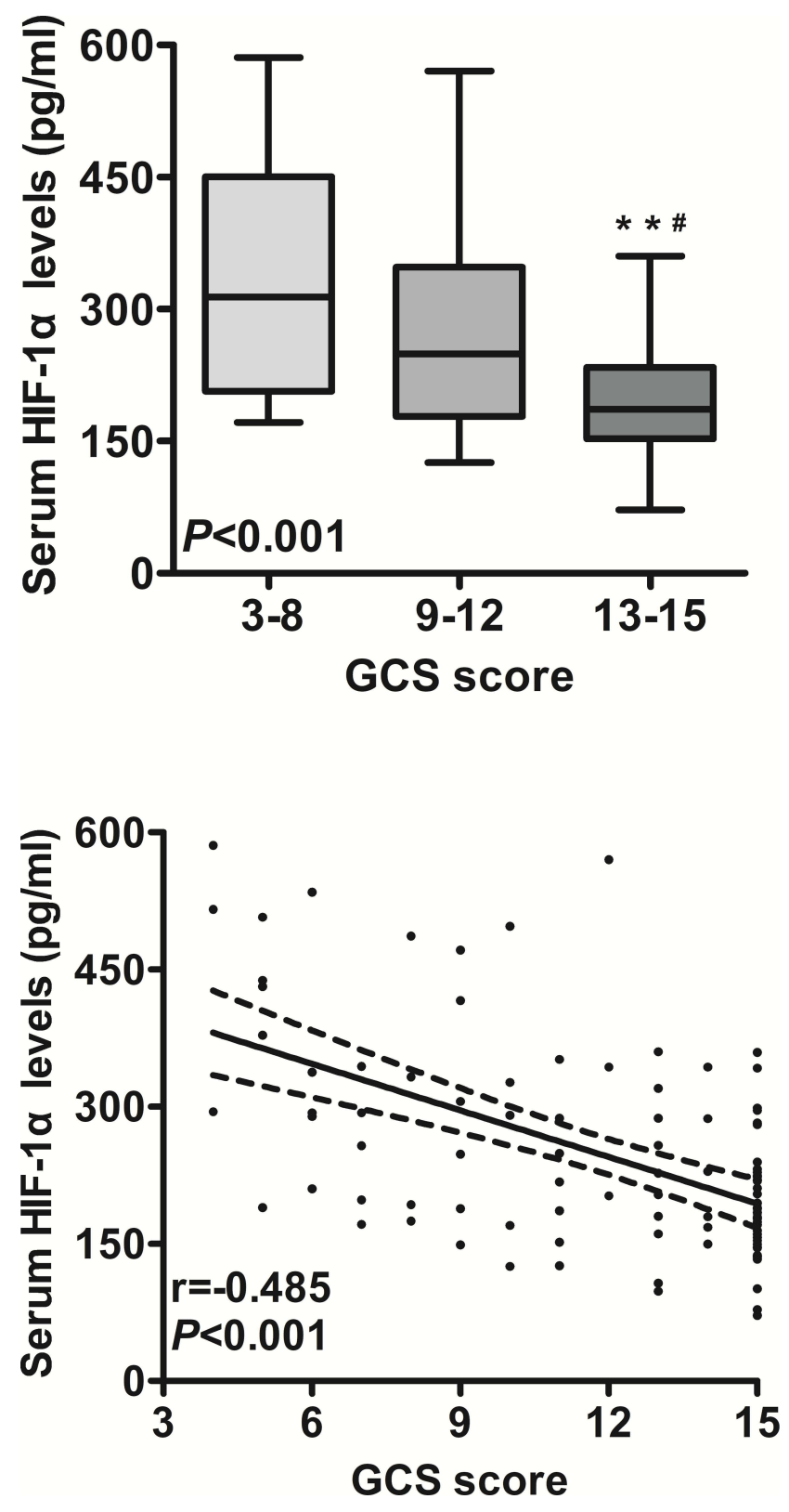

Figure 2 Relationship between serum hypoxia-inducible factor Ialpha levels and Glasgow Coma Scale score among intracerebral hemorrhage patients. When Glasgow Coma Scale score was a continuous variable, serum hypoxia-inducible factor Ialpha levels were significantly raised with decreased Glasgow Coma Scale score using Spearman correlation coefficients $(P<0.001)$. And, Glasgow Coma Scale score was identified as a categorical variable and subsequently, patients were divided into three groups in accordance with Glasgow Coma Scale score, namely, 3-8, 9-12 and 13-15, and therefore patients with Glasgow Coma Scale score 3-8 had substantially highest serum hypoxiainducible factor Ialpha levels, followed by Glasgow Coma Scale score 9-12 and then 1315 using Kruskal-Wallis $H$-test $(P<0.00 \mathrm{I})$. In boxplot, ${ }^{* * P}<0.0 \mathrm{I}$ compared with Glasgow Coma Scale score 3-8, and ${ }^{\#} P<0.05$ compared with Glasgow Coma Scale score 9-12. In correlation graph, the solid line means line of best fit and dashed line represents $95 \%$ confidence interval of a population mean.

Abbreviations: GCS, Glasgow Coma Scale; HIF-I $\alpha$, hypoxia-inducible factor Ialpha.

acute ischemic stroke in a small sample size of 40 patients, ${ }^{18}$ this has been the first study to match serum HIF$1 \alpha$ levels to hemorrhagic severity and long-term outcomes in 

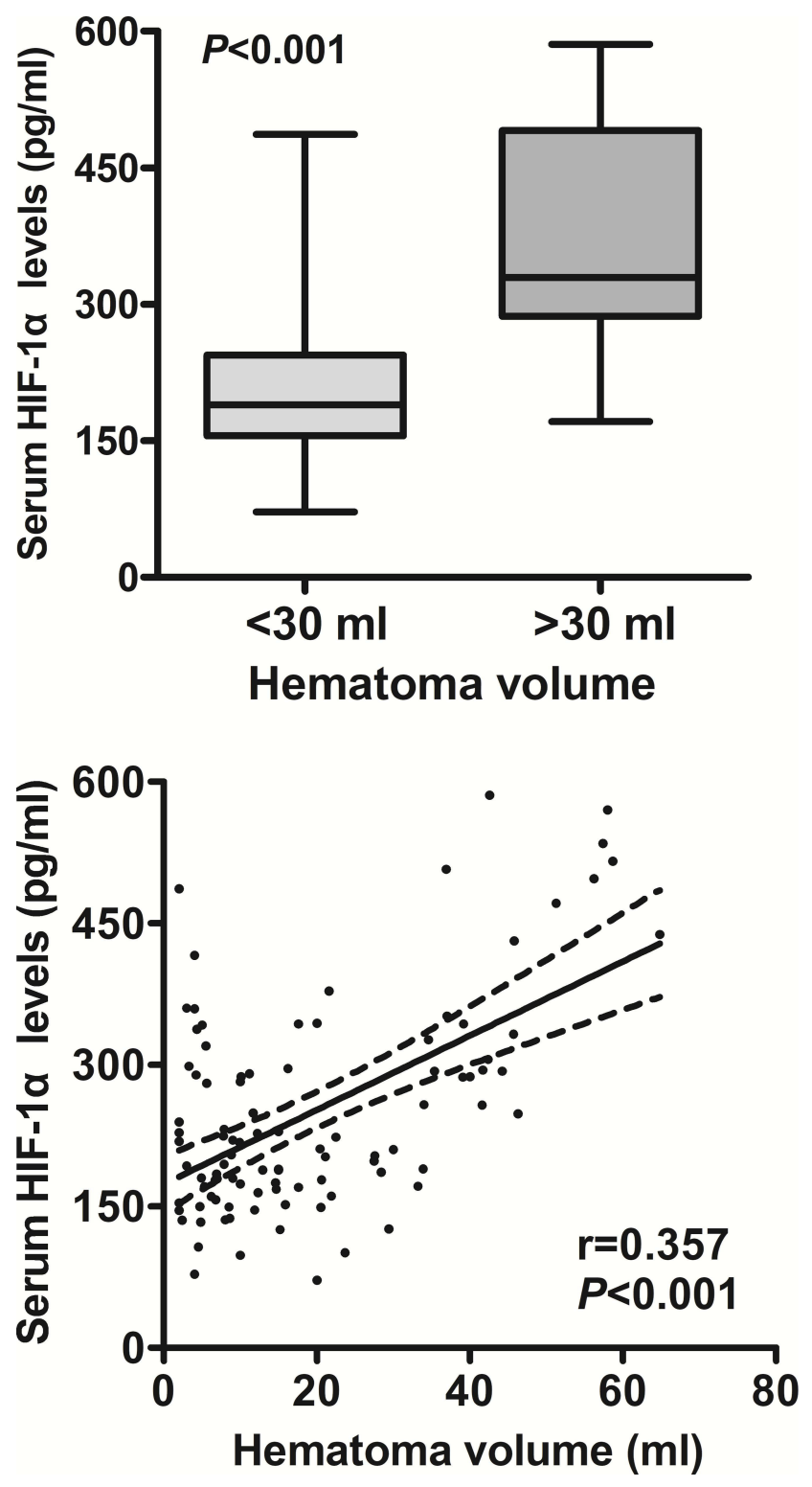

Figure 3 Relationship between serum hypoxia-inducible factor Ialpha levels and hematoma volume after intracerebral hemorrhage. When hematoma volume was a continuous variable, serum hypoxia-inducible factor lalpha levels were significantly raised with rising hematoma volume using Spearman correlation coefficients $(P<0.00 \mathrm{I})$. When hematoma volume was identified as a categorical variable and subsequently, patients were divided into two groups in accordance with hematoma volume, namely, above $30 \mathrm{~mL}$ and below $30 \mathrm{~mL}$, patients with hematoma volume above $30 \mathrm{~mL}$ had substantially higher serum hypoxia-inducible factor Ialpha levels than those with hematoma volume below $30 \mathrm{~mL}$ using Mann-Whitney U-test $(P<0.00 \mathrm{I})$. In correlation graph, the solid line means line of best fit and dashed line represents $95 \%$ confidence interval of a population mean.

Abbreviation: HIF-I $\alpha$, hypoxia-inducible factor Ialpha.

patients after ICH. The results of this study verified that serum HIF-1 $\alpha$ level, as a continuous variable, was linked to hemorrhagic severity and, as a categorical variable, was independently associated with 90-day unfavorable outcome in ICH patients. Thus, serum HIF- $1 \alpha$ might have the potential to be a promising biomarker for aiding in severity assessment and prognostic prediction after hemorrhagic stroke.
Table 2 Correlative Analysis Between Serum Hypoxia Inducible Factor-lalpha Levels and Other Variables Using Multivariate Linear Regression Analysis in Acute Intracerebral Hemorrhage

\begin{tabular}{|l|c|c|}
\hline Components & $\boldsymbol{t}$ & P value \\
\hline Age (years) & 1.529 & 0.130 \\
Sex (male/female) & 0.600 & 0.550 \\
Hypertension & 0.841 & 0.403 \\
Diabetes mellitus & 1.592 & 0.115 \\
Current smoking & -1.480 & 0.142 \\
Alcohol consumption & 1.703 & 0.092 \\
Admission time (h) & -0.946 & 0.347 \\
Glasgow Coma Scale score & -2.552 & 0.012 \\
Hematoma volume (mL) & 3.182 & 0.002 \\
Blood-collection time (h) & 0.663 & 0.509 \\
\hline
\end{tabular}

Note: Correlations were determined using multivariate linear regression analysis.

HIF-1 $\alpha$ is an upstream transcription factor, which is induced by hypoxia. ${ }^{5}$ It regulates the subsequent expression of many kinds of proteins responding to the various pathophysiological conditions induced by hypoxia. ${ }^{6}$ In vitro study showed that, in rat cultured cortical neurons deprived of oxygen and glucose, activation of HIF-1 $\alpha$ was induced rapidly and transiently in a dose- and timedependent manner. ${ }^{20}$ In rats with permanent middle cerebral artery occlusion, a temporal and spatial induction of HIF- $1 \alpha$ messenger RNA was found in the penumbra. ${ }^{21}$ Upregulation of HIF-1 $\alpha$ protein was revealed in perihematomal tissues of $\mathrm{ICH}$ rats, as well as thrombin and erythrocyte lysate was implicated in HIF-1 $\alpha$ upregulation through inhibiting HIF- $1 \alpha$ degradation. ${ }^{10}$ In line with the preceding data, ${ }^{10}$ a significant elevation of serum HIF-1 $\alpha$ levels was revealed in this cohort of ICH patients.

HIF-1 $\alpha$, a transcription factor that translocates from the cytosol to the nucleus upon activation, is involved in pathologic conditions such as hypoxia or ischemia. ${ }^{22}$ Its expression was upregulated in neurons around hematoma in rats with acute $\mathrm{ICH} .{ }^{10}$ There are only modest and transient reductions in cerebral blood flow after ICH. ${ }^{23}$ Whether the resultant hypoxia is sufficient to induce HIF-1 $\alpha$ is uncertain. However, in cultured smooth-muscle cells, thrombin (ie, a nonhypoxic mechanism) can upregulate HIF- $1 \alpha{ }^{24}$ Clearly, thrombin contributes to brain injury after $\mathrm{ICH}^{25}$ Thrombin was involved in HIF-1 $\alpha$ upregulation through reducing HIF-1 $\alpha$ degradation, ${ }^{10}$ suggesting another mechanism by which ICH might induce HIF-1 $\alpha$. However, HIF$1 \alpha$, acting as a transcription factor, is located in intracellular compartment. ${ }^{8,9}$ Thus, it is speculated that the 

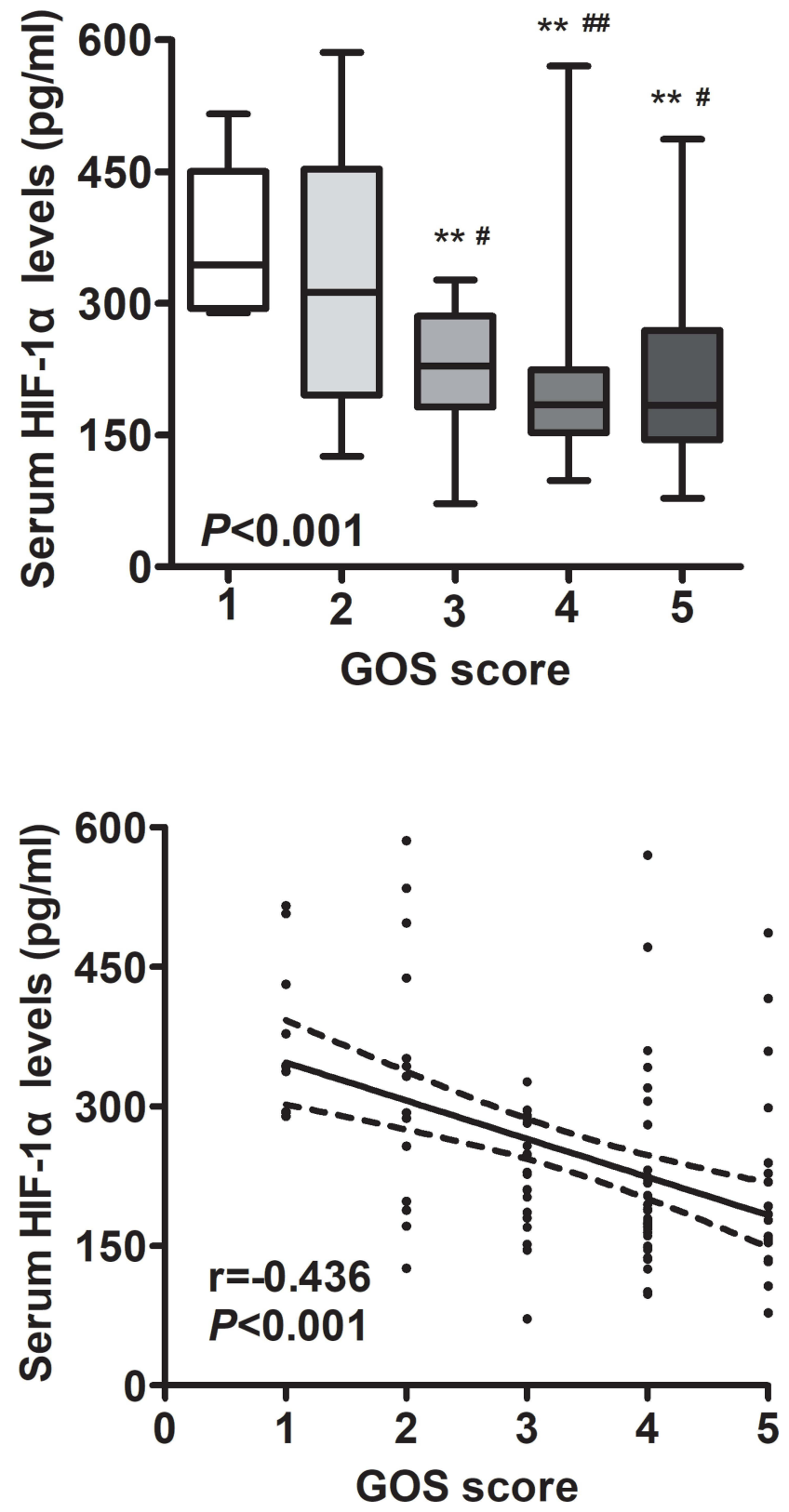

Figure 4 Relationship between serum hypoxia-inducible factor lalpha levels and Glasgow Outcome Scale score at 90 days after intracerebral hemorrhage. Whether Glasgow Outcome Scale score was a continuous or categorical variable, serum hypoxia-inducible factor Ialpha levels were significantly declined with raised Glasgow Outcome Scale score using Spearman correlation coefficients $(P<0.00 \mathrm{I})$ or Kruskal-Wallis $H$-test $(P<0.00 I)$. In boxplot, $* * P<0.01$ compared with Glasgow Outcome Scale score I, as well as ${ }^{\#} P<0.05$ and ${ }^{\# \#} P<0.01$ compared with Glasgow Outcome Scale score 2. In correlation graph, the solid line means line of best fit and dashed line represents $95 \%$ confidence interval of a population mean.

Abbreviations: GOS, Glasgow Outcome Scale; HIF-I $\alpha$, hypoxia-inducible factor Ialpha.

destruction of neurons might lead to the release of HIF-1 $\alpha$ into peripheral blood via disrupted bloodbrain barrier after ICH. Overall, HIF-1 $\alpha$ in peripheral blood of this cohort of patients might be at least partly derived from brain tissues injured by hemorrhage.
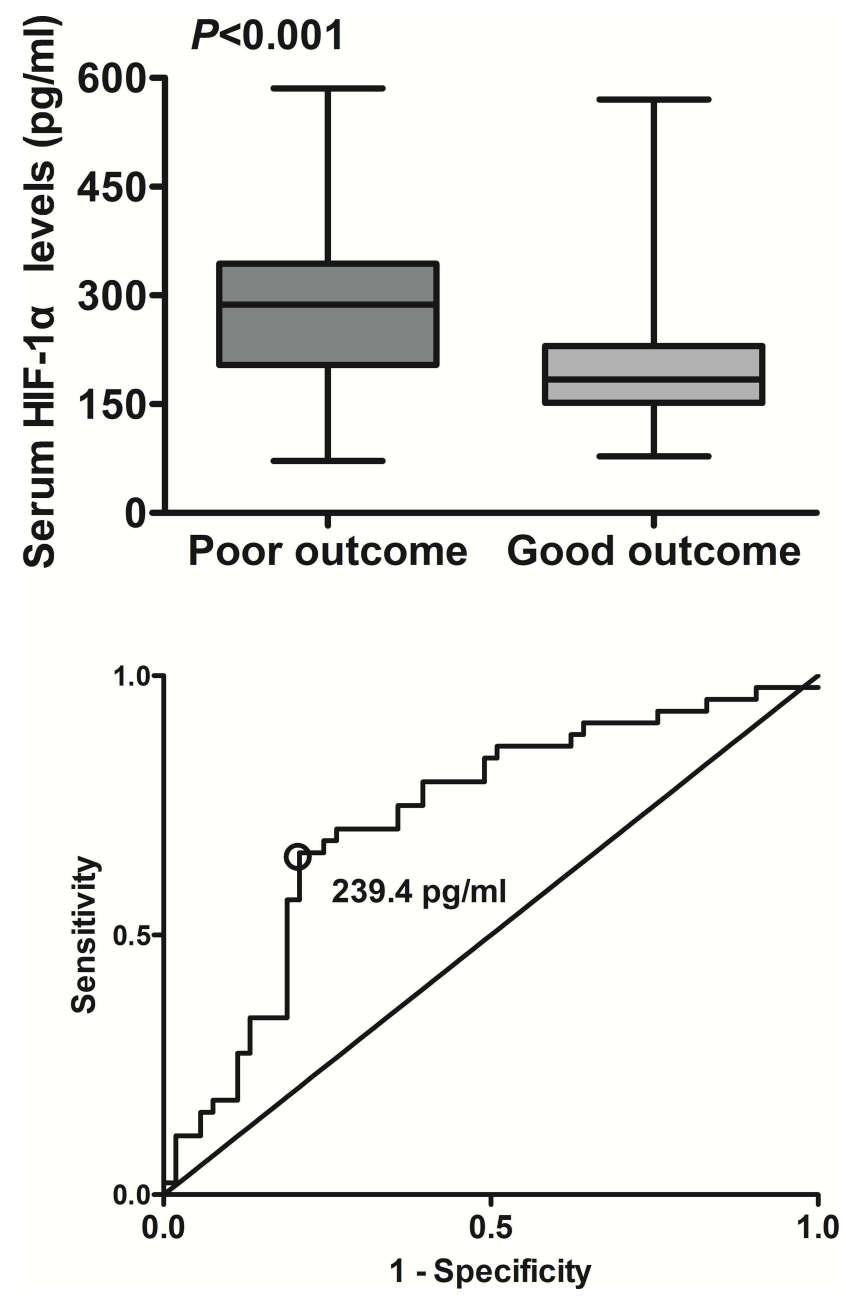

Figure 5 Relationship between serum hypoxia-inducible factor Ialpha levels and poor outcome at 90 days after intracerebral hemorrhage. A poor outcome was defined as Glasgow Outcome Scale of $\mathrm{I}-3$ at 90 days after hemorrhagic stroke. Serum hypoxia-inducible factor Ialpha levels were significantly elevated in patients with a poor outcome, as compared to those with a good outcome using MannWhitney $U$-test $(P<0.00 \mathrm{I})$. Under receiver-operating characteristic curve, serum hypoxia-inducible factor Ialpha levels remarkably predicted post-stroke 90-day poor outcome (area under curve, 0.725; 95\% confidence interval, 0.625-0.81I); and serum hypoxia-inducible factor Ialpha levels more than $239.4 \mathrm{pg} / \mathrm{mL}$ distinguished patients with development of poor 90-day outcome with specificity and sensitivity values of $79.3 \%$ and $65.9 \%$ (Youden index J, 0.452 ) respectively.

Abbreviations: GOS, Glasgow Outcome Scale; HIF-I $\alpha$, hypoxia-inducible factor Ialpha.

HIF-1 $\alpha$ plays an essential role in the fate of injured neurons. ${ }^{11-13}$ HIF-1 $\alpha$ appears to be involved in the cell's response to acute brain injury with a double-edged sword effect. $^{26}$ Previous studies demonstrated that HIF-1 $\alpha$ mediated the death of cultured cells deprived of oxygen and/or glucose, ${ }^{27,28}$ whereas HIF-1 $\alpha$-induced angiogenesis and glycolytic metabolism might be important for cells to survive under hypoxic/ischemic condition. ${ }^{29,30}$ In neuronspecific HIF-1 $\alpha$ knock-out mice with middle cerebral artery occlusion, tissue damage was increased and the 


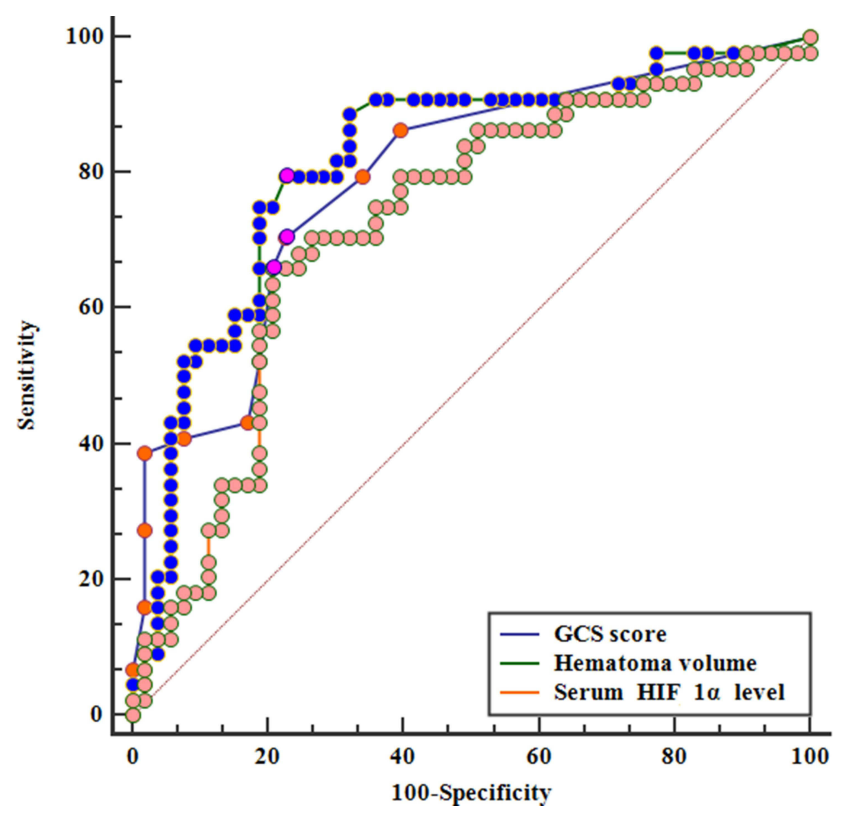

Figure 6 Comparison of discriminatory capability with respect to serum hypoxiainducible factor Ialpha level, Glasgow Coma Scale score and hematoma volume for 90-day poor outcome following acute intracerebral hemorrhage under receiver operating characteristic curve. A poor outcome was referred to as Glasgow Outcome Scale of I-3 at 90 days after hemorrhagic stroke. Prognostic predictive ability of serum hypoxia-inducible factor Ialpha level (area under curve, 0.725; 95\% confidence interval, $0.625-0.8 \mathrm{II}$ ) was similar to those of Glasgow Coma Scale score (area under curve, 0.792; 95\% confidence interval, 0.697-0.868; $P=0.248$ ) and hematoma volume (area under curve, $0.817 ; 95 \%$ confidence interval, 0.725-0.888; $P=0.124)$.

Abbreviations: GCS, Glasgow Coma Scale; HIF-I $\alpha$, hypoxia-inducible factor Ialpha.

survival rate was reduced. $^{31}$ In contrast, in another a similar model with knock-out of HIF-1 $\alpha$, ischemic damage was decreased. ${ }^{32}$ Moreover, some inconsistent results also existed in other studies. ${ }^{11-17}$ In summary, the effect of HIF-1 $\alpha$ on brain function is unclear and understanding its role in brain injury will undoubtedly provide potential approaches to treat acute brain injury diseases including ICH. However, in 40 patients with acute ischemic stroke, elevated serum HIF-1 $\alpha$ concentration was correlated with cerebral infarct size. ${ }^{18}$ Thus, the relationship between serum HIF-1 $\alpha$ concentration and severity of brain injury may not be dependent on the function of HIF-1 $\alpha$. In this way, our study also found the similar result that, whether using univariate or multivariate analysis, serum HIF-1 $\alpha$ levels of ICH patients were closely correlated with baseline GCS score and hematoma volume, both of which were shown as quantitative and qualitative variables. Taken together, serum HIF-1 $\alpha$ levels may be able to reflect the extent of hemorrhagic severity.

To my best knowledge, it remains unclear whether serum HIF-1 $\alpha$ levels are associated with prognosis of some acute brain injury diseases including $\mathrm{ICH}$. On the one hand, our study showed that serum HIF-1 $\alpha$ levels of ICH patients were highly correlated with GOS score, and displayed a high discriminatory capability for 90-day poor prognosis defined as GOS score 1-3; moreover, its prognostic predictive ability was in range of GCS score and hematoma volume. In other words, serum HIF-1 $\alpha$ levels might have a strong power to predict long-term poor prognosis after ICH. On the other hand, serum HIF-1 $\alpha$ levels as a categorical variable, GCS score and hematoma volume were all independently associated with 90-day poor prognosis. In a word, serum HIF-1 $\alpha$ may emerge as a promising prognostic biomarker for $\mathrm{ICH}$.

In our study, it was found that serum HIF-1 $\alpha$ levels were highly correlated with clinical outcome, GCS score and hematoma volumes assessed by head CT scan. However, the average turnaround time for laboratory results in the emergency departments is between 30 and 40 minutes currently. The uninterpreted head computed tomographic images are available to be viewed within 10 minutes. The GCS score is immediately apparent upon physical examination of the patient. Although measurement of circulating HIF-1 $\alpha$ may currently not add much to the armamentariums of the clinician, it holds great potential, as it were, to become more time efficient and, more importantly, it may be able to provide more detailed data to predict outcome. Spontaneous intracranial hemorrhage is a catastrophic event with poor prognosis. ${ }^{1}$ Therefore, to have another way to predict outcome will not only help the clinicians but also the family to make decisions as well as necessary adjustments to care for the recovering patient.

\section{Conclusions}

This study was designed to discern the utility of the serum HIF-1 $\alpha$ levels in the prediction of long-term prognosis and assessment of ICH severity. To the best of my knowledge, this is the first series to show that serum HIF-1 $\alpha$ levels are remarkably correlated with the severity and poor prognosis after ICH. Thus, serum HIF-1 $\alpha$ may be of clinical value for aiding in both evaluation of the $\mathrm{ICH}$ status and prediction of the $\mathrm{ICH}$ prognosis. 
Table 3 Demographic, Clinical, Radiological and Biochemical Factors for 90-Day Poor Outcome in Acute Intracerebral Hemorrhage

\begin{tabular}{|c|c|c|c|}
\hline \multirow{2}{*}{ Components } & \multicolumn{2}{|c|}{ 90-Day Poor Outcome } & \multirow[t]{2}{*}{$P$ value } \\
\hline & Presence & Absence & \\
\hline Number & $44(45.4 \%)$ & $53(54.6 \%)$ & \\
\hline Age (years) & $67(56-77)$ & $56(46-66)$ & 0.002 \\
\hline Sex (male/female) & $25 / 19$ & $30 / 23$ & 0.983 \\
\hline Hypertension & $27(61.4 \%)$ & $30(56.6 \%)$ & 0.635 \\
\hline Diabetes mellitus & $5(11.4 \%)$ & $9(17.0 \%)$ & 0.433 \\
\hline Current smoking & $\mathrm{II}(25.0 \%)$ & $12(22.6 \%)$ & 0.786 \\
\hline Alcohol consumption & $12(27.3 \%)$ & $20(37.7 \%)$ & 0.275 \\
\hline Admission time $(\mathrm{h})$ & $5.9(4.0-8.0)$ & $7.0(4.0-12.0)$ & 0.268 \\
\hline Systolic arterial pressure $(\mathrm{mmHg})$ & $155(144-176)$ & $157(143-170)$ & 0.656 \\
\hline Diastolic arterial pressure $(\mathrm{mmHg})$ & $89(80-102)$ & $88(78-98)$ & 0.329 \\
\hline Lobar hemorrhage & $10(22.7 \%)$ & $10(18.9 \%)$ & 0.640 \\
\hline Infratentorial hemorrhage & $7(15.9 \%)$ & $8(15.1 \%)$ & 0.912 \\
\hline Intraventricular hemorrhage & $\mid 4(3 \mid .8 \%)$ & $12(22.6 \%)$ & 0.310 \\
\hline GCS score & $10(6-13)$ & $15(13-15)$ & $<0.001$ \\
\hline Hematoma volume $(\mathrm{mL})$ & $28.9(15.5-40.8)$ & $7.9(4.7-14.5$ & $<0.001$ \\
\hline Blood-collection time (h) & $8.0(5.0-10.5)$ & $9.0(6.0-14.0)$ & 0.150 \\
\hline Blood leucocyte count $\left(\times 10^{9} / \mathrm{l}\right)$ & $9.8(6.8-12.0)$ & $8.7(6.9-10.2)$ & 0.190 \\
\hline Serum glucose levels $(\mathrm{mmol} / \mathrm{l})$ & $7.5(6.1-9.6)$ & $6.0(4.9-8.0)$ & 0.006 \\
\hline Serum HIF-I $\alpha>239.4 \mathrm{pg} / \mathrm{mL}$ & $29(65.9 \%)$ & $12(22.6 \%)$ & $<0.001$ \\
\hline
\end{tabular}

Notes: Quantitative data were reported as medians with 25th-75th percentiles. Qualitative data were presented as counts (proportions). Intergroup comparisons of various variables were performed using the $\chi^{2}$ test or Fisher's exact test for qualitative data, and Mann-Whitney U-test for quantitative data. Poor outcome was designated as Glasgow Outcome Scale score of I-3.

Abbreviations: GCS, Glasgow Coma Scale; HIF-I $\alpha$, hypoxia-inducible factor-Ialpha.

Table 4 Predictive Factors of 90-Day Poor Outcome Among Patients with Acute Intracerebral Hemorrhage Using Univariable Logistic Regression Analysis

\begin{tabular}{|c|c|c|c|}
\hline Components & Odds Ratio & $95 \% \mathrm{Cl}$ & P value \\
\hline Age (years) & 1.048 & $1.014-1.083$ & 0.005 \\
\hline Sex (male/female) & 0.991 & $0.442-2.221$ & 0.983 \\
\hline Hypertension & 1.218 & $0.539-2.749$ & 0.636 \\
\hline Diabetes mellitus & 0.627 & $0.194-2.030$ & 0.436 \\
\hline Current smoking & 1.139 & $0.446-2.909$ & 0.786 \\
\hline Alcohol consumption & 0.619 & $0.260-1.470$ & 0.277 \\
\hline Admission time $(\mathrm{h})$ & 0.931 & $0.862-1.006$ & 0.072 \\
\hline Systolic arterial pressure $(\mathrm{mmHg})$ & 1.008 & $0.992-1.024$ & 0.357 \\
\hline Diastolic arterial pressure $(\mathrm{mmHg})$ & 1.019 & $0.995-1.043$ & 0.127 \\
\hline Lobar hemorrhage & 1.265 & $0.472-3.387$ & 0.640 \\
\hline Infratentorial hemorrhage & 1.064 & $0.353-3.209$ & 0.912 \\
\hline Intraventricular hemorrhage & 1.594 & $0.646-3.935$ & 0.312 \\
\hline GCS score & 0.718 & $0.620-0.831$ & $<0.001$ \\
\hline Hematoma volume (mL) & 1.086 & $1.047-1.126$ & $<0.001$ \\
\hline Blood-collection time $(\mathrm{h})$ & 0.944 & $0.882-1.010$ & 0.094 \\
\hline Blood leucocyte count $\left(\times 10^{9} / \mathrm{l}\right)$ & 1.109 & $0.98 \mathrm{I}-\mathrm{I} .254$ & 0.098 \\
\hline Serum glucose levels (mmol/l) & 1.161 & $1.007-1.339$ & 0.040 \\
\hline Serum HIF-I $\alpha>239.4 \mathrm{pg} / \mathrm{mL}$ & 6.606 & $2.697-16.178$ & 0.001 \\
\hline
\end{tabular}

Note: Results were presented as odds ratio ( $95 \%$ confidence interval) using the univariate binary logistic regression analysis. Abbreviations: $95 \% \mathrm{Cl}$, 95\% confidence interval; GCS, Glasgow Coma Scale; HIF-I $\alpha$, hypoxia inducible factor-Ialpha. 
Table 5 Multivariate Logistic Regression Analysis for Risk Factors of Poor Outcome in Acute Intracerebral Hemorrhage

\begin{tabular}{|l|c|c|}
\hline Variables & Odds Ratio (95\% Confidence Interval) & P value \\
\hline Age (years) & $1.018(0.978-1.059)$ & 0.382 \\
Glasgow Coma Scale score & $0.837(0.701-0.998)$ & 0.048 \\
Hematoma volume $(\mathrm{mL})$ & $1.050(1.004-1.098)$ & 0.032 \\
Serum glucose levels $(\mathrm{mmol} / \mathrm{l})$ & $0.854(0.659-1.105)$ & 0.229 \\
Serum HIF- $\mid \alpha$ level $>239.4 \mathrm{pg} / \mathrm{mL}$ & $5.133(1.117-23.593)$ & 0.036 \\
\hline
\end{tabular}

Note: Results were presented as odds ratio ( $95 \%$ confidence interval) using the binary logistic regression analysis.

\section{Abbreviations}

AUC, area under the curve; GCS, Glasgow Coma Scale; GOS, Glasgow Outcome Scale; HIF-1 $\alpha$, hypoxia-inducible factor 1alpha; ICH, intracerebral hemorrhage; ROC, receiver operating characteristic.

\section{Data Sharing Statement}

The data that support the findings of this study are available on request from the corresponding author. The data are not publicly available due to privacy or ethical restrictions.

\section{Ethical Review Approval}

The study was approved by the medical ethics committee of the Affiliated Hangzhou First People's Hospital, Zhejiang University School of Medicine, Opinion Number: [2020] Medical Ethics Review No. (058)-01. All procedures were conducted in compliance with the guidelines of the Declaration of Helsinki.

\section{Informed Consent}

The written informed consent was acquired from controls themselves or relatives of patients.

\section{Acknowledgments}

The authors thank all participants for their willingness to participate in this study.

\section{Disclosure}

The authors declare that they have no competing interests.

\section{References}

1. Aguilar MI, Freeman WD. Spontaneous intracerebral hemorrhage. Semin Neurol. 2010;30(05):555-564. doi:10.1055/s-0030-1268865

2. Nath FP, Jenkins A, Mendelow AD, Graham DI, Teasdale GM. Early hemodynamic changes in experimental intracerebral hemorrhage. J Neurosurg. 1986;65(5):697-703. doi:10.3171/jns.1986.65.5.0697

3. Nath FP, Kelly PT, Jenkins A, Mendelow AD, Graham DI, Teasdale GM. Effects of experimental intracerebral hemorrhage on blood flow, capillary permeability, and histochemistry. J Neurosurg. 1987;66(4):555-562. doi:10.3171/jns.1987.66.4.0555
4. Senn R, Elkind MS, Montaner J, Christ-Crain M, Katan M. Potential role of blood biomarkers in the management of nontraumatic intracerebral hemorrhage. Cerebrovasc Dis. 2014;38:395-409. doi:10.1159/000366470

5. Ke Q, Costa M. Hypoxia-inducible factor-1 (HIF-1). Mol Pharmacol. 2006;70(5):1469-1480. doi:10.1124/mol.106.027029

6. Koyasu S, Kobayashi M, Goto Y, Hiraoka M, Harada H. Regulatory mechanisms of hypoxia-inducible factor 1 activity: two decades of knowledge. Cancer Sci. 2018;109(3):560-571. doi:10.1111/ cas. 13483

7. Semenza GL. Defining the role of hypoxia-inducible factor 1 in cancer biology and therapeutics. Oncogene. 2010;29(5):625-634. doi:10.1038/onc.2009.441

8. Ramamoorthy P, Shi H. Ischemia induces different levels of hypoxia inducible factor- $1 \alpha$ protein expression in interneurons and pyramidal neurons. Acta Neuropathol Commun. 2014;2(1):51. doi:10.1186/ 2051-5960-2-51

9. Ramamoorthy P, Xu G, Shi H. Expression of hypoxia inducible factor 1alpha is protein kinase A-dependent in primary cortical astrocytes exposed to severe hypoxia. Neurochem Res. 2019;44(1):258-268. doi:10.1007/s11064-018-2516-9

10. Jiang Y, Wu J, Keep RF, Hua Y, Hoff JT, Xi G. Hypoxia-inducible factor-1alpha accumulation in the brain after experimental intracerebral hemorrhage. J Cereb Blood Flow Metab. 2002;22(6):689-696. doi:10.1097/00004647-200206000-00007

11. Ding JY, Kreipke CW, Speirs SL, Schafer P, Schafer S, Rafols JA. Hypoxia-inducible factor-1alpha signaling in aquaporin upregulation after traumatic brain injury. Neurosci Lett. 2009;453(1):68-72. doi:10.1016/j.neulet.2009.01.077

12. Higashida T, Peng C, Li J, et al. Hypoxia-inducible factor- $1 \alpha$ contributes to brain edema after stroke by regulating aquaporins and glycerol distribution in brain. Curr Neurovasc Res. 2011;8 (1):44-51. doi:10.2174/156720211794520251

13. Wang Z, Meng CJ, Shen XM, et al. Potential contribution of hypoxiainducible factor- $1 \alpha$, aquaporin- 4 , and matrix metalloproteinase- 9 to blood-brain barrier disruption and brain edema after experimental subarachnoid hemorrhage. J Mol Neurosci. 2012;48(1):273-280. doi:10.1007/s12031-012-9769-6

14. Li YQ, Hui ZR, Tao T, et al. Protective effect of hypoxia inducible factor-1 $\alpha$ gene therapy using recombinant adenovirus in cerebral ischaemia-reperfusion injuries in rats. Pharm Biol. 2020;58 (1):438-446. doi:10.1080/13880209.2020.1762667

15. Lv B, Li F, Fang J, et al. Hypoxia inducible factor $1 \alpha$ promotes survival of mesenchymal stem cells under hypoxia. Am J Transl Res. 2017;9(3):1521-1529.

16. Zhu T, Zhan L, Liang D, et al. Hypoxia-inducible factor $1 \alpha$ mediates neuroprotection of hypoxic postconditioning against global cerebral ischemia. $J$ Neuropathol Exp Neurol. 2014;73(10):975-986. doi:10.1097/NEN.0000000000000118

17. Huang T, Huang W, Zhang Z, et al. Hypoxia-inducible factor- $1 \alpha$ upregulation in microglia following hypoxia protects against ischemia-induced cerebral infarction. Neuroreport. 2014;25 (14):1122-1128. doi:10.1097/WNR.0000000000000236 
18. Xue L, Chen H, Lu K, Huang J, Duan H, Zhao Y. Clinical significance of changes in serum neuroglobin and HIF-1 $\alpha$ concentrations during the early-phase of acute ischemic stroke. J Neurol Sci. 2017;375:52-57. doi:10.1016/j.jns.2017.01.039

19. Kothari RU, Brott T, Broderick JP, et al. The ABCs of measuring intracerebral hemorrhage volumes. Stroke. 1996;27(8):1304-1305. doi:10.1161/01.STR.27.8.1304

20. Ruscher K, Isaev N, Trendelenburg G, et al. Induction of hypoxia inducible factor 1 by oxygen glucose deprivation is attenuated by hypoxic preconditioning in rat cultured neurons. Neurosci Lett. 1998;254(2):117-120. doi:10.1016/S0304-3940(98)00688-0

21. Bergeron M, Yu AY, Solway KE, Semenza GL, Sharp FR. Induction of hypoxia-inducible factor-1 (HIF-1) and its target genes following focal ischaemia in rat brain. Eur J Neurosci. 1999;11(12):4159-4170. doi:10.1046/j.1460-9568.1999.00845.x

22. Kallio PJ, Okamoto K, O'Brien S, et al. Signal transduction in hypoxic cells: inducible nuclear translocation and recruitment of the $\mathrm{CBP} / \mathrm{p} 300$ coactivator by the hypoxia-inducible factor-1alpha. $E M B O$ J. 1998;17(22):6573-6586. doi:10.1093/emboj/17.22.6573

23. Patel TR, Schielke GP, Hoff JT, Keep RF, Lorris Betz A. Comparison of cerebral blood flow and injury following intracerebral and subdural hematoma in the rat. Brain Res. 1999;829(1-2):125-133. doi:10.1016/S0006-8993(99)01378-5

24. Richard DE, Berra E, Pouyssegur J. Nonhypoxic pathway mediates the induction of hypoxia-inducible factor 1alpha in vascular smooth muscle cells. J Biol Chem. 2000;275(35):26765-26771. doi:10.1016/ S0021-9258(19)61441-9

25. Lee KR, Betz AL, Keep RF, Chenevert TL, Kim S, Hoff JT. Intracerebral infusion of thrombin as a cause of brain edema. J Neurosurg. 1995;83(6):1045-1050. doi:10.3171/jns.1995.83.6.1045
26. Shi H. Hypoxia inducible factor 1 as a therapeutic target in ischemic stroke. Curr Med Chem. 2009;16(34):4593-4600. doi:10.2174/ 092986709789760779

27. Carmeliet P, Dor Y, Herbert JM, et al. Role of HIF-1alpha in hypoxia-mediated apoptosis, cell proliferation and tumour angiogenesis. Nature. 1998;394(6692):485-490. doi:10.1038/28867

28. Halterman MW, Miller CC, Federoff HJ. Hypoxia-inducible factor-1alpha mediates hypoxia-induced delayed neuronal death that involves p53. J Neurosci. 1999;19(16):6818-6824. doi:10.1523/ JNEUROSCI.19-16-06818.1999

29. Bergeron M, Gidday JM, Yu AY, Semenza GL, Ferriero DM, Sharp FR. Role of hypoxia-inducible factor-1 in hypoxia-induced ischemic tolerance in neonatal rat brain. Ann Neurol. 2000;48 (3):285-296. doi:10.1002/1531-8249(200009)48:3<285::AIDANA2>3.0.CO;2-8

30. Semenza GL. Surviving ischemia: adaptive responses mediated by hypoxia-inducible factor 1. J Clin Invest. 2000;106(7):809-812. doi:10.1172/JCI11223

31. Baranova O, Miranda LF, Pichiule P, Dragatsis I, Johnson RS, Chavez JC. Neuron-specific inactivation of the hypoxia inducible factor $1 \alpha$ increases brain injury in a mouse model of transient focal cerebral ischemia. $J$ Neurosci. 2007;23(23):6320-6332. doi:10.1523/ JNEUROSCI.0449-07.2007

32. Helton R, Cui J, Scheel JR, et al. Brain-specific knock-out of hypoxia-inducible factor- $1 \alpha$ reduces rather than increases hypoxicischemic damage. J Neurosci. 2005;16(16):4099-4107. doi:10.1523/ JNEUROSCI.4555-04.2005
Therapeutics and Clinical Risk Management

\section{Publish your work in this journal}

Therapeutics and Clinical Risk Management is an international, peerreviewed journal of clinical therapeutics and risk management, focusing on concise rapid reporting of clinical studies in all therapeutic areas, outcomes, safety, and programs for the effective, safe, and sustained use of medicines. This journal is indexed on PubMed Central, CAS,

\section{Dovepress}

EMBase, Scopus and the Elsevier Bibliographic databases. The manuscript management system is completely online and includes a very quick and fair peer-review system, which is all easy to use. Visit http://www.dovepress.com/testimonials.php to read real quotes from published authors. 